\title{
Convergent and Divergent Solutions of Singular Partial Differential Equations with Resonance or Small Denominators
}

\author{
By
}

Masafumi Yoshino*

\begin{abstract}
We show the solvability and nonsolvability of a singular nonlinear system of partial differential equations with resonance in a class of functions holomorphic in some neighborhood of the origin. These results are applied to the normal form theory of a singular vector field.
\end{abstract}

\section{$\S 1 . \quad$ Introduction}

In this paper, we study the solvability and nonsolvability of a singular nonlinear system of partial differential equations which appear in the normal form theory of vector fields. It is well-known that under the Poincaré condition or a Diophantine condition the sytem of equations has a convergent power series solution locally. (cf. Remark 2.) We are interested in the solvability in a class of convergent power series without any Diophantine condition although there are infinite resonances or small denominators. We are also interested in the divergence caused by the presence of a nontrivial Jordan block in the linear part. Because the singular operator which we consider has infinite resonance

Communicated by T. Kawai. Received May 15, 2006. Revised October 6, 2006, November $30,2006$.

2000 Mathematics Subject Classification(s): Primary 35C10; Secondary 37F50, 37G05.

Key words and phrases: solvability, resonance, small denominators, divergence, convergence.

Partially supported by Grant-in-Aid for Scientific Research (No. 11640183), Ministry of Education, Science and Culture, Japan.

* Department of Mathematics, Graduate School of Science, Hiroshima University, 1-3-1 Kagamiyama, Higashi-hiroshima, Hiroshima 739-8526, Japan.

e-mail: yoshino@math.sci.hiroshima-u.ac.jp

(c) 2007 Research Institute for Mathematical Sciences, Kyoto University. All rights reserved. 
or small denominators, a standard energy method or an iterative method does not work due to the presence of high loss of derivatives.

To our best knowledge, few results are known for such operators. One interesting approach for the problem is the geometric viewpoint. To be more precise, let us consider an equation appearing from a Hamiltonian vector field. Clearly, the normalizing transformation satisfies an equation with infinite resonance. (cf. (2.2).) It is well-known that the formal power series solutions of the equation do not converge in general. Due to Ito and Zung, the convergence is equivalent to the existence of a certain number of integrals. (cf. [1] and [5].)

We shall give a rather simple wide class of nonlinear perturbations for which one can always find a convergent solution, which is different from an integrability condition because we put no restriction on the resonance dimension. (cf. [1] and [5].) We also construct a Liouville type linear part and a nonlinear perturbation for which a divergence of a (unique) solution occurs. (cf. Proposition 3.1.) This especially shows that our sufficient condition of a nonlinear perturbation is necessary in general.

We are also interested in the divergence phenomenon caused by the presence of a nontrivial Jordan block of the linear part in a Siegel case. In fact, if this is the case, then the solutions corresponding to the normalizing transformation generally diverge even if we assume a Diophantine condition. (cf. Proposition 3.2.) Theorem 2.1 also gives a convergence criterion for these operators.

This paper is organized as follows. In Section 2, we state convergence results. In Section 3, we study the divergence and Diophantine phenomena. In Section 4 , we prepare necessary lemmas. In Section 5 we prove our theorem.

\section{$\S 2 . \quad$ Convergence Criterions}

Let $x={ }^{t}\left(x_{1}, \ldots, x_{n}\right) \in \mathbb{C}^{n}, n \geq 2$ be the variable in $\mathbb{C}^{n}$, and $\mathbb{R}$ be the set of real numbers. Let $\Lambda$ be an $n$-square constant matrix. Let $L_{\Lambda}$ be the Lie derivative of the linear vector field $\Lambda x \cdot \partial_{x}$

$$
L_{\Lambda} v=[\Lambda x, v]=\left\langle\Lambda x, \partial_{x} v\right\rangle-\Lambda v,
$$

where $\left\langle\Lambda x, \partial_{x} v\right\rangle=\sum_{j=1}^{n}(\Lambda x)_{j}\left(\partial / \partial x_{j}\right) v$, with $(\Lambda x)_{j}$ being the $j$-th component of $\Lambda x$. We consider the system of equations

$$
L_{\Lambda} u=R(u(x))
$$

where $u={ }^{t}\left(u_{1}, u_{2}, \ldots, u_{n}\right)$ is an unknown vector function and

$$
R(x)={ }^{t}\left(R_{1}(x), R_{2}(x), \ldots, R_{n}(x)\right)
$$


is holomorphic in some neighborhood of $x=0$ in $\mathbb{C}^{n}$ such that $R(x)=O\left(|x|^{2}\right)$ when $|x| \rightarrow 0$. The equation (2.2) appears as a linearizing equation of a singular vector field. (cf. [4]). Because we can always reduce $\Lambda$ to a Jordan normal form by the linear change of the unknown functions $U=A u$, we may assume that $\Lambda$ is put in a Jordan normal form. Moreover we assume that there exists $\exists \tau_{0}$, $0 \leq \tau_{0} \leq \pi$ such that

$$
\text { every component of } e^{-i \tau_{0}} \Lambda \text { is a real number. }
$$

It follows that if $\lambda_{j}(j=1,2, \ldots, n)$ are the eigenvalues of $\Lambda$ with multiplicity, then we have

$$
\exists \tau_{0}, 0 \leq \tau_{0} \leq \pi, e^{-i \tau_{0}} \lambda_{j} \in \mathbb{R} \quad(j=1,2, \ldots, n) .
$$

If we set $u(x)=x+v(x), v(x)=O\left(|x|^{2}\right)$, then $v$ satisfies the following equation

$$
L_{\Lambda} v=R(x+v(x))
$$

Let $\mathbb{Z}_{+}$be the set of nonnegative integers, and let $\mathbb{Z}_{+}^{n}(k)(k \geq 0)$ be the $n$-product of $\mathbb{Z}_{+}, \gamma={ }^{t}\left(\gamma_{1}, \gamma_{2}, \ldots, \gamma_{n}\right)$ such that $|\gamma|=\gamma_{1}+\gamma_{2}+\cdots+\gamma_{n} \geq k$. For $\gamma \in \mathbb{Z}_{+}^{n}$, we set $x^{\gamma}=x_{1}^{\gamma_{1}} \cdots x_{n}^{\gamma_{n}}$. For $k \geq 0$ and $n \geq 1$, we denote by $\mathbb{C}_{k}^{n}[[x]]$ the set of formal power series $\sum_{|\eta| \geq k} u_{\eta} x^{\eta}\left(u_{\eta} \in \mathbb{C}^{n}\right)$. We also define the convergent $n$-vector power series which vanishes up to the $(k-1)$-th derivative by $\mathbb{C}_{k}^{n}[x]$. We decompose $\Lambda=\Lambda_{S}+\Lambda_{N}$, where $\Lambda_{S}$ and $\Lambda_{N}$ are the semi-simple and the nilpotent part of $\Lambda$, respectively. We denote by $L_{\Lambda_{S}}$ the Lie derivative of the linear vector field $\Lambda_{S} x \cdot \partial_{x}$.

For a formal power series $f(x)=\sum_{\gamma} f_{\gamma} x^{\gamma}$, we define the majorant of $f$, $M(f)(x)$ by

$$
M(f):=\sum_{\gamma}\left|f_{\gamma}\right| x^{\gamma}
$$

For a vector $f=\left(f_{1}, f_{2}, \ldots, f_{n}\right)$ we define $M(f):=\left(M\left(f_{1}\right), M\left(f_{2}\right), \ldots\right.$, $\left.M\left(f_{n}\right)\right)$. For a formal power series with real coefficients $a(x)=\sum_{\gamma} a_{\gamma} x^{\gamma}$ and $b(x)=\sum_{\gamma} b_{\gamma} x^{\gamma}$, we define $a \ll b$ if $a_{\gamma} \leq b_{\gamma}$ for all $\gamma \in \mathbb{Z}_{+}^{n}$. We define

$$
\left(f_{1}(x), f_{2}(x), \ldots, f_{n}(x)\right) \ll\left(g_{1}(x), g_{2}(x), \ldots, g_{n}(x)\right)
$$

if $f_{j}(x) \ll g_{j}(x)$ for $j=1,2, \ldots, n$.

Let $c>0$ be a constant. Let $\mathcal{A}_{+}\left(\right.$resp. $\left.\mathcal{A}_{-}\right)$be the set of $g(x)=$ ${ }^{t}\left(g_{1}(x), \ldots, g_{n}(x)\right) \in \mathbb{C}_{2}^{n}[x]$ such that

$$
\left(L_{\Lambda_{S}}-c\right) M(g) \gg 0 \quad\left(\text { resp. } \quad\left(L_{\Lambda_{S}}+c\right) M(g) \ll 0\right)
$$


and that $g(x)$ is a finite sum of the functions $f={ }^{t}\left(f_{1}, f_{2}, \ldots, f_{n}\right) \in \mathbb{C}_{2}^{n}[x]$ with the following expansion at the origin

$$
f_{j}(x)=x_{\nu} \sum_{\gamma} f_{j, \gamma} x^{\gamma}, \quad f_{j, \gamma} \in \mathbb{C}, \quad j=1,2, \ldots, n,
$$

where $\nu$ is such that the $j$-th and the $\nu$-th components of $\Lambda_{S}$ belong to the same Jordan block of $\Lambda_{N}$. We can prove that $\mathcal{A}_{ \pm}$are linear spaces. (cf. Lemma 4.3.) Then we have

Theorem 2.1. $\quad$ Suppose that (2.3) holds. Let $R(x) \in \mathcal{A}_{ \pm}$. Assume that $R(x)$ is a polynomial with degree $<c+1$ if $\Lambda_{N} \neq 0$. Then, (2.5) has a holomorphic solution in some neighborhood of the origin $x=0$.

Remark 1. If we drop the conditions of Theorem 2.1, then we encounter the divergence caused by small denominators and the presence of a Jordan block. More precisely, we have

(a) If $c=0$, then Theorem 2.1 does not hold in general because of small denominators. Namely, the condition $L_{\Lambda_{S}} M(R) \gg 0$ or $L_{\Lambda_{S}} M(R) \ll 0$ is not sufficient. (cf. Proposition 3.1.)

(b) There exists $R$ such that neither $L_{\Lambda_{S}} M(R) \ll 0$ nor $L_{\Lambda_{S}} M(R) \gg 0$ holds for which Theorem 2.1 does not hold. This follows from Proposition 3.1 in view of the arbitrariness of $R^{\prime}$ in Proposition 3.1.

(c) If $\Lambda$ is not semi-simple, then there exists $R$ which is not a polynomial such that Theorem 2.1 does not hold. This follows from Proposition 3.2.

Finally we note that because (2.5) has infinite resonance in general, the uniqueness of a solution in Theorem 2.1 does not hold in general.

Remark 2. We will briefly review the notions used in this paper. Let $\lambda_{j}(j=1,2, \ldots, n)$ be the eigenvalues of $\Lambda$ with multiplicity. We say that $\lambda_{j}(j=1,2, \ldots, n)$ satisfy the Poincaré condition if the convex hull of $\lambda_{j}$ $(j=1,2, \ldots, n)$ in the complex plane does not contain the origin $0 \in \mathbb{C}$. We can easily see that the Poincaré condition is equivalent to the following estimate: there exist $C>0$ and $K>0$ independent of $\alpha$ such that

$$
\left|\langle\lambda, \alpha\rangle-\lambda_{j}\right| \geq C|\alpha|, \quad \forall \alpha=\left(\alpha_{1}, \alpha_{2}, \ldots, \alpha_{n}\right) \in \mathbb{Z}_{+}^{n},|\alpha| \geq K,
$$

for $j=1,2, \ldots, n$, where $\lambda=\left(\lambda_{1}, \lambda_{2}, \ldots, \lambda_{n}\right)$ and $\langle\lambda, \alpha\rangle=\sum_{i=1}^{n} \lambda_{i} \alpha_{i}$.

For $\alpha=\left(\alpha_{1}, \alpha_{2}, \ldots, \alpha_{n}\right) \in \mathbb{Z}^{n}$ we define $\|\alpha\|=\sum_{i=1}^{n}\left|\alpha_{i}\right|$. We say that $\lambda \in \mathbb{R}^{n}$ is a Diophantine vector if there exist $\exists \tau>0$ and $\exists C>0$ such that

$$
\left|\langle\lambda, \alpha\rangle-\lambda_{j}\right| \geq C\|\alpha\|^{-n-\tau}, \quad \forall \alpha \in \mathbb{Z}^{n},\|\alpha\| \geq 2, j=1,2, \ldots, n .
$$


We call (2.10) a Diophantine condition. The vector which does not satisfy a Diophantine condition is called a Liouville vector. By considering the special case of (2.10) we have the classical definition of a Diophantine number. We say that a number $t \in \mathbb{R} \backslash \mathbb{Q}$ is a Diophantine number if there exist $\tau>0$ and $C>0$ such that for every $p, q \in \mathbb{Z}, q>0$, one has $\left|t-\frac{p}{q}\right| \geq C q^{-2-\tau}$. The Liouville numbers are the complement of Diophantine numbers in $\mathbb{R} \backslash \mathbb{Q}$.

Finally, we give the definition of a Brjuno type Diophantine condition. We set $\ell_{j}(\alpha)=\langle\lambda, \alpha\rangle-\lambda_{j}$ and define

$$
\omega_{k}=\inf \left\{\left|\ell_{j}(\alpha)\right| ; \ell_{j}(\alpha) \neq 0, \alpha \in \mathbb{Z}^{n}, 2 \leq\|\alpha\| \leq 2^{k}, j=1,2, \ldots, n\right\},
$$

where $k=1,2, \ldots$. Then we say that $\lambda$ satisfies the Brjuno type Diophantine condition if

$$
-\sum_{k \geq 0} \frac{\ln \left(\omega_{k+1}\right)}{2^{k}}<+\infty .
$$

We can easily see that a Diophantine vector satisfies (2.12).

\section{§3. Divergence and Diophantine Phenomena}

In this section we study divergence caused by small denominators and the presence of a Jordan block. We consider in $x \in \mathbb{C}^{2}$

$$
L_{\Lambda} u=R(x+u), \quad \Lambda=\left(\begin{array}{cc}
1 & 0 \\
0 & -\tau
\end{array}\right),
$$

where $\tau>0$ is a Liouville number chosen later and $u=O\left(|x|^{2}\right)$. Then we have

Proposition 3.1. For every $R^{\prime}(x)$ holomorphic in some neighborhood of the origin, there exist a Liouville number $\tau>0$ and a holomorphic perturbation $R^{\prime \prime}(x) \not \equiv 0$ such that $L_{\Lambda_{S}} M\left(R^{\prime \prime}\right) \gg 0$ or $L_{\Lambda_{S}} M\left(R^{\prime \prime}\right) \ll 0$ holds and that the unique formal power series solution of (3.1) with $R=R^{\prime}+R^{\prime \prime}$ diverges.

Proof. We construct an irrational number $\tau$ by the continued fraction expansion $\tau=\left[a_{1}, a_{2}, \ldots\right], a_{j} \in \mathbb{N}$. Namely, if we define the sequence $\left\{p_{n}\right\}$ and $\left\{q_{n}\right\}$ by

$$
\begin{aligned}
& p_{n}=a_{n} p_{n-1}+p_{n-2}, \quad n \geq 2, p_{0}=0, p_{1}=1, \\
& q_{n}=a_{n} q_{n-1}+q_{n-2}, \quad n \geq 2, q_{0}=1, q_{1}=a_{1},
\end{aligned}
$$


then we have $p_{n} / q_{n} \rightarrow \tau(n \rightarrow \infty)$. Moreover, we have (cf. [3])

$$
\frac{1}{\left(a_{n+1}+2\right) q_{n}^{2}}<\left|\tau-\frac{p_{n}}{q_{n}}\right|<\frac{1}{a_{n+1} q_{n}^{2}}, \quad n \geq 0 .
$$

We substitute the expansion of $u=\left(u_{1}, u_{2}\right)$ and $R_{j}(x+u)$,

$$
\begin{aligned}
u_{j} & =\sum_{|\eta| \geq 2} u_{j, \eta} x^{\eta} \\
R_{j}(x+u) & =\sum_{|\gamma| \geq 2} R_{j, \gamma}\left(x_{1}+\sum u_{1, \eta} x^{\eta}\right)^{\gamma_{1}}\left(x_{2}+\sum u_{2, \eta} x^{\eta}\right)^{\gamma_{2}}
\end{aligned}
$$

into the equation (3.1). Then we have the recurrence relations

$$
\begin{aligned}
& \left(\eta_{1}-\tau \eta_{2}-1\right) u_{1, \eta}=R_{1, \eta}+P_{\eta, 1}\left(u_{j, \delta},|\delta|<|\eta|, j=1,2\right), \\
& \left(\eta_{1}-\tau \eta_{2}+\tau\right) u_{2, \eta}=R_{2, \eta}+P_{\eta, 2}\left(u_{j, \delta},|\delta|<|\eta|, j=1,2\right),
\end{aligned}
$$

where $P_{\eta, j}$ is a polynomial of $u_{k, \delta}$ 's with coefficients given by the expansions of $R$. Now suppose that $a_{1}, a_{2}, \ldots, a_{n}$ are given. We determine $p_{n}$ and $q_{n}$ by (3.2) and (3.3), and we want to determine $u_{1, \eta}$ with $\eta=\left(p_{n}+1, q_{n}\right)$ from (3.5) assuming that $u_{j, \delta}(|\delta|<|\eta|, j=1,2)$ are already determined. This is possible if $\tau$ avoids a finite number of rational points. If the absolute value of $P_{\eta, 1}\left(u_{j, \delta},|\delta|<|\eta|, j=1,2\right)$ is smaller than $2^{|\eta|+1}$, then we take $R_{1, \eta}$ such that $\left|R_{1, \eta}\right|=2 \cdot 3^{|\eta|}$. On the other hand, if the absolute value of $P_{\eta, 1}\left(u_{j, \delta},|\delta|<\right.$ $|\eta|, j=1,2)$ is larger than $2^{|\eta|+1}$, then we take $R_{1, \eta}$ such that $\left|R_{1, \eta}\right|=2^{|\eta|}$. It follows that the absolute value of the right-hand side of (3.5) is larger than $2^{|\eta|}$. In view of (3.4), we determine $a_{n+1}$ such that $\left|u_{1,\left(p_{n}+1, q_{n}\right)}\right| \geq\left(p_{n}+q_{n}\right)$ !. This is possible if we take $a_{n+1}$ sufficiently large. Moreover, by the definition of continued fractions we see that the approximant $\left[a_{1}, a_{2}, \ldots, a_{n+1}\right]$ avoids the finite number of rational points given in the above if we take $a_{n+1}$ sufficiently large. Next we determine $p_{n+1}$ and $q_{n+1}$ from (3.2) and (3.3). Then we want to determine $u_{1, \eta}$ with $\eta=\left(p_{n+1}+1, q_{n+1}\right)$ from (3.5). We can determine the terms $u_{j, \delta}(|\delta|<|\eta|, j=1,2)$ if $\tau$ avoids a finite number of rational points. This is possible if $a_{n+2}$ is sufficiently large. Then we repeat the same argument as in the above. Clearly, $R(x)$ is holomorphic in some neighborhood of the origin. On the other hand, if we take $a_{n+1}$ so that $a_{n+1}$ is larger than polynomial order of $q_{n}$, then it follows from (3.4) that $\tau$ is a Liouville number. Therefore we can determine a Liouville number $\tau$ so that we have a divergent formal power series solution $u=\sum_{|\eta| \geq 2} u_{\eta} x^{\eta}$.

By the definition of a continued fraction expansion, we have $p_{n}+1-\tau q_{n}-$ $1>0$ or $p_{n}+1-\tau q_{n}-1<0$ according as $n$ is odd or even. For simplicity, 
we take $p_{n}$ and $q_{n}$ for odd $n$. If we take $R_{1, \eta}^{\prime \prime}$ appropriately, then we have $R_{1, \eta} \neq 0$ and the support of $R_{1, \eta}^{\prime \prime}$ is contained in $\eta_{1}-\tau \eta_{2}-1>0$. This proves that $L_{\Lambda_{S}} M\left(R^{\prime \prime}\right) \gg 0$. By a similar argument we can also treat the case $L_{\Lambda_{S}} M\left(R^{\prime \prime}\right) \ll 0$. This completes the proof.

Remark 3. We know that almost all nonlinear perturbations of a Liouville type linear operator has a divergent solution. (cf. [2]) Our result shows that for any nonlinear perturbation there exists a Liouville number $\tau$ such that the divergence occurs if we add a limited type of nonlinear perturbations.

Next we study the divergence caused by the presence of a nontrivial Jordan block even if a Diophantine condition is verified. We consider in $x \in \mathbb{C}^{3}$

$$
L_{\Lambda} u=R(x+u), \quad \Lambda=\left(\begin{array}{ccc}
1 & 0 & 0 \\
0 & -\tau & -1 \\
0 & 0 & -\tau
\end{array}\right)
$$

where $\tau>0$ is an irrational number and $u=O\left(|x|^{2}\right)$. Then we have

Proposition 3.2. Let $c>0$. For every irrational number $\tau>0$ there exists $R \not \equiv 0$ which is not a polynomial such that $\left(L_{\Lambda_{S}}-c\right) M(R) \gg 0$ or $\left(L_{\Lambda_{S}}+c\right) M(R) \ll 0$ holds and that the unique formal power series solution of (3.7) diverges.

Proof. Let $K$ be such that $K>c+2$. We denote by $[c]$ the largest integer which does not exceed $c$. Then we define

$$
R_{1}(x)=x_{1}^{[c]+2} \tilde{R}_{1}\left(x_{1}\right), \quad R_{2}(x)=\sum_{\max \{c, 2\} \leq i-\tau j<K} x_{1}^{i} x_{2}^{j}, \quad R_{3}(x) \equiv 0,
$$

where $\tilde{R}_{1}$ is holomorphic at the origin such that $\tilde{R}_{1} \gg 0$. We can easily see that $\left(L_{\Lambda_{S}}-c\right) M(R) \gg 0$.

We will construct the solution $u=\left(u_{1}, u_{2}, u_{3}\right)$ of (3.7). We set $u_{1}(x)=$ $x_{1} w_{1}\left(x_{1}\right)$. Then it follows from the first equation of (3.7) that $w_{1}$ satisfies $x_{1} \partial_{1} w_{1}=x_{1}^{[c]+1}\left(1+w_{1}\right)^{[c]+2} \tilde{R}_{1}\left(x_{1}\left(1+w_{1}\right)\right)$. By the elementary computations, we can easily show that the equation has a holomorphic solution $w_{1}\left(x_{1}\right)$ such that $w_{1} \gg 0$. Next $u_{3}$ satisfies $\left(x_{1} \partial_{1}-\tau x_{2} \partial_{2}-\tau x_{3} \partial_{3}-x_{3} \partial_{2}+\tau\right) u_{3}=0$. By the irrationality of $\tau$, we have $u_{3}=0$.

Next, by the second equation of (3.7) $u_{2}$ satisfies

$$
\left(x_{1} \partial_{1}-\tau x_{2} \partial_{2}-\tau x_{3} \partial_{3}-x_{3} \partial_{2}+\tau\right) u_{2}=R_{2}\left(x_{1}\left(1+w_{1}\right), x_{2}+u_{2}\right) .
$$


We denote by $g(x)$ the right-hand side of (3.9). By the expansions $u_{2}(x)=$ $\sum_{\alpha} u_{2, \alpha} x^{\alpha}$ and $g(x)=\sum_{\alpha} g_{\alpha} x^{\alpha}$, we define the vectors $U$ and $G$ by

$$
U:={ }^{t}\left(u_{2,\left(\alpha_{1}, N-\ell, \ell\right)}\right)_{\ell=0}^{N}, G:={ }^{t}\left(g_{\left(\alpha_{1}, N-\ell, \ell\right)}\right)_{\ell=0}^{N},
$$

where we may assume that $N+\alpha_{1} \geq 2, N, \alpha_{1} \in \mathbb{Z}_{+}$. Indeed, by (3.8) and (3.9) one may assume that the order of $g(x)$ is greater than 2. Hence in the definition of $G$ in (3.10) we may assume that $N+\alpha_{1} \geq 2, N, \alpha_{1} \in \mathbb{Z}_{+}$. On the other hand, because the differential operator in the left-hand side of (3.9) preserves homogeneous polynomials, we may assume the conditions for $U$. By substituting the expansions of $g(x)$ and $u_{2}(x)$ into (3.9), we have

$$
\left(\alpha_{1}-\tau N+\tau\right) U-\mathcal{M}_{N} U=G
$$

where $\mathcal{M}_{N}$ is given by

$$
\mathcal{M}_{N}=\left(\begin{array}{ccccccc}
0 & 0 & 0 & \ldots & 0 & 0 & 0 \\
N & 0 & 0 & \ldots & 0 & 0 & 0 \\
0 & N-1 & 0 & \ldots & 0 & 0 & 0 \\
0 & 0 & N-2 & \ldots & 0 & 0 & 0 \\
\vdots & \vdots & \vdots & \ddots & \vdots & \vdots & \vdots \\
0 & 0 & 0 & \ldots & 2 & 0 & 0 \\
0 & 0 & 0 & \ldots & 0 & 1 & 0
\end{array}\right), \quad N \geq 1
$$

and $\mathcal{M}_{0}=0$. By inductive arguments we get

$$
u_{2,\left(\alpha_{1}, N-\ell, \ell\right)}=\sum_{r=0}^{\ell} \frac{1}{\left(\alpha_{1}-\tau N+\tau\right)^{r+1}} \frac{(N-\ell+r) !}{(N-\ell) !} g_{\left(\alpha_{1}, N-\ell+r, \ell-r\right)}
$$

for $\ell=0,1, \ldots, N$, because $\alpha_{1}-\tau N+\tau \neq 0$ by the assumption $\alpha_{1}+N \geq 2$ and the irrationality of $\tau$.

We set $\mathcal{L}:=x_{1} \partial_{1}-\tau x_{2} \partial_{2}-\tau x_{3} \partial_{3}$. By the definition of $R_{2}$, we obtain $(\mathcal{L}-c) M\left(R_{2}\right) \gg 0$. Because the order of $x_{1} w_{1}, u_{2}$ or $R_{2}$ is equal to or greater than 2 by the constructions of $w_{1}$ and $u_{2}$ or the definition of $R_{2}$, it follows that, in the Taylor expansion of the right-hand side of (3.9) the terms $x^{\alpha}$ $\left(\alpha_{1}+N=2, \alpha_{2}+\alpha_{3}=N\right)$ appear only in the expansion of $R_{2}\left(x_{1}, x_{2}\right)$. Hence, we see that $(\mathcal{L}-c) M\left(u_{2}\right) \gg 0$ up to the terms of order 2 . Now, suppose that $(\mathcal{L}-c) M\left(u_{2}\right) \gg 0$ holds up to order $k$. Then, we want to show that $(\mathcal{L}-c) M(g) \gg 0$ holds up to order at least $k+1$. Indeed, in view of the definition of $g$ we may consider the terms of order less than or equal to $k+1$ which appear in $x_{1}^{i}\left(1+w_{1}\right)^{i}\left(x_{2}+u_{2}\right)^{j}$. Let us consider the term $\left(\begin{array}{l}i \\ \nu\end{array}\right)\left(\begin{array}{l}j \\ \mu\end{array}\right) x_{1}^{i} w_{1}^{\nu} x_{2}^{j-\mu} u_{2}^{\mu},(\nu \geq 0$ 
and $0 \leq \mu \leq j)$. Then, by the definition of $g$ we have $(\mathcal{L}-c) M\left(x_{1}^{i} x_{2}^{j-\mu}\right) \gg 0$ because $i-\tau(j-\mu)>i-\tau j \geq c$. On the other hand the terms of order less than or equal to $k+1$ appearing in $u_{2}^{\mu}$ satisfies $(\mathcal{L}-c) M(\cdot) \gg 0$. Similarly we have $(\mathcal{L}-c) M\left(w_{1}^{\nu}\right) \gg 0$. Hence we have the assertion. It follows from (3.9) that $(\mathcal{L}-c) M\left(u_{2}\right) \gg 0$ holds up to order at least $k+1$. By induction, we obtain $(\mathcal{L}-c) M\left(u_{2}\right) \gg 0$. Because $\tau>0$, it follows that $(\mathcal{L}+\tau-c) M\left(u_{2}\right) \gg$ $(\mathcal{L}-c) M\left(u_{2}\right) \gg 0$. On the other hand, we have that $(\mathcal{L}-1-c) M\left(u_{1}\right) \gg 0$, because the order of $u_{1}=u_{1}\left(x_{1}\right)$ is greater than $[c]+2$ by the construction. We also have $(\mathcal{L}+\tau-c) M\left(u_{3}\right)=0 \gg 0$. Therefore we have $\left(L_{\Lambda_{S}}-c\right) M(u) \gg 0$.

Next we will show that $u_{2} \gg 0$. Indeed, by the relation $(\mathcal{L}-c) M\left(u_{2}\right) \gg 0$ we see that the support of the Taylor expansion of $u_{2}$ satisfies that $\alpha_{1}-\tau \alpha_{2}-$ $\tau \alpha_{3} \geq c>0$. Because $R \gg 0$, it follows from (3.13) that the coefficients in Taylor expansion of $u_{2}$ of homogeneous order 2 are nonnegative. Namely, we have $u_{2} \gg 0$ up to order 2 . Hence, we have $g(x) \gg 0$ up to at least order 3 , because $R \gg 0$. It follows from (3.13) that $u_{2} \gg 0$ up to at least order 3. By inductive argument, we have $u_{2} \gg 0$.

We will show the divergence. By the definition of $g$, we can write $g_{\alpha}=$ $\tilde{g}_{\alpha}+h_{\alpha}$, where $\tilde{g}_{\alpha}$ comes from $R_{2}(x)$ and $h_{\alpha}$ comes from terms containing $w_{1}$ and $u_{2}$. By the assumption and what we have proved in the above, we have $\tilde{g}_{\alpha} \geq 0$ and $h_{\alpha} \geq 0$. Because $\tilde{g}_{\left(\alpha_{1}, N, 0\right)}=1$, it follows from (3.13) that $u_{2,\left(\alpha_{1}, 0, N\right)} \geq N !\left(\alpha_{1}-\tau N+\tau\right)^{-N-1}$. Hence $u_{2}$ diverges. This ends the proof.

\section{$\S 4 . \quad$ Preliminary Lemmas}

In order to prove lemmas, we use subspaces of $\mathcal{A}_{ \pm}$. Let $f \in \mathcal{A}_{-}$be given by (2.8). For $\rho>0$, we introduce the norm of $f$ by

$$
\|f\|_{\rho}:=\sum_{j=1}^{n} M\left(f_{j}\right)(\rho, \ldots, \rho)=\sum_{j=1}^{n} \sum_{\gamma}\left|f_{j, \gamma}\right| \rho^{|\gamma|+1},
$$

if the right-hand side is finite. The set of all $f$ such that $\|f\|_{\rho}<\infty$ is denoted by $\mathcal{A}_{-, \rho}$. We similarly define $\mathcal{A}_{+, \rho}$. If we make the change of the variables $x_{j} \mapsto \varepsilon x_{j}$, then we may assume that $\rho>1$ in the above definition. Hence we assume $\rho>1$ in the following. For the sake of simplicity, we sometimes omit the suffix of the norm $\|\cdot\|_{\rho}$, and denote it by $\|\cdot\|$ if there is no fear of confusion.

Let the operators $Q_{ \pm}$on the spaces $\mathcal{A}_{\mp}$ be defined by

$$
Q_{ \pm} V(x)=-\int_{0}^{ \pm \infty} e^{-t \Lambda} V\left(e^{t \Lambda} x\right) d t, \quad V \in \mathcal{A}_{\mp},
$$

if the right-hand side integral converges. We denote by $\mathcal{A}_{\mp}^{0}$ the subset of elements of $\mathcal{A}_{\mp}$ which are polynomials in $x$. Then we have 
Lemma 4.1. Suppose that (2.3) holds. Moreover, assume that $\Lambda_{N}=0$. Then, $Q_{ \pm}$is a continuous linear operator on $\mathcal{A}_{\mp}$ into $\mathcal{A}_{\mp}$ such that $L_{\Lambda} Q_{ \pm} V=$ $V$ for every $V \in \mathcal{A}_{\mp}$. Moreover, there exists $c_{1}>0$ such that $\left\|Q_{ \pm} V\right\|_{\rho} \leq$ $c_{1}\|V\|_{\rho}$ for all $V \in \mathcal{A}_{\mp}$. If $\Lambda_{N} \neq 0$, then $Q_{ \pm}$is a linear operator on $\mathcal{A}_{\mp}^{0}$ into $\mathcal{A}_{\mp}^{0}$.

Proof. Because the proof is similar, we prove the lemma for $Q_{+}$. By multiplying (2.5) with $e^{-i \tau_{0}}$, we may assume that all components of $\Lambda$ are real. For $V(x)={ }^{t}\left(V_{1}(x), V_{2}(x), \ldots, V_{n}(x)\right) \in \mathcal{A}_{-}$, let $V_{j}(x)=\sum_{\gamma} x^{\gamma} V_{j, \gamma}$ $(j=1,2, \ldots, n)$ be the Taylor expansion of $V_{j}(x)$. We set $\lambda={ }^{t}\left(\lambda_{1}, \lambda_{2}, \ldots, \lambda_{n}\right)$. We write $\Lambda=\Lambda_{S}+\Lambda_{N}$, where $\Lambda_{S}$ and $\Lambda_{N}$ are the semi-simple and the nilpotent parts of $\Lambda$, respectively. Because $\left[\Lambda_{S}, \Lambda_{N}\right]=0$, we have

$$
e^{-t \Lambda} V\left(e^{t \Lambda} x\right)=e^{-t \Lambda_{N}} e^{-t \Lambda_{S}} V\left(e^{t \Lambda} x\right)
$$

Since $\left(e^{t \Lambda} x\right)^{\gamma}=\left(e^{t \Lambda_{N}} x\right)^{\gamma} e^{t\langle\lambda, \gamma\rangle}$, it follows that the $j$-th component of $e^{-t \Lambda_{S}} V\left(e^{t \Lambda} x\right)$ is given by

$$
\sum_{\gamma} e^{-t \lambda_{j}}\left(e^{t \Lambda} x\right)^{\gamma} V_{j, \gamma}=\sum_{\gamma}\left(e^{t \Lambda_{N}} x\right)^{\gamma} e^{t\langle\lambda, \gamma\rangle-t \lambda_{j}} V_{j, \gamma}
$$

On the other hand, it follows from (2.7) that, for every $\gamma \in \mathbb{Z}_{+}^{n}(2)$ and $j=$ $1,2, \ldots, n$, we have $\langle\lambda, \gamma\rangle-\lambda_{j} \leq-c<0$ if $V_{j, \gamma} \neq 0$. Hence we obtain

$$
\exp \left(t\langle\lambda, \gamma\rangle-t \lambda_{j}\right) \leq e^{-c t}, \quad \forall t \geq 0 .
$$

It follows that for each $t \geq 0$, the sum (4.4) converges.

If $\Lambda$ is semi-simple, i.e., $\Lambda_{N}=0$, then it follows from (4.4) and (4.5) that the integral (4.2) converges. If $\Lambda_{N} \neq 0$, then we see that the growth of terms appearing in $\left(e^{t \Lambda_{N}} x\right)^{\gamma}$ is at most $t^{|\gamma|(\ell-1)}$, where $\ell \geq 2$ is the maximal size of the Jordan block of $\Lambda_{N}$. Because we assume that $V$ is a polynomial in case $\Lambda_{N} \neq 0$, it follows from (4.3), (4.4) and (4.5) that the integral in (4.2) converges and it is a polynomial of $x$.

Because the substitution $x^{\gamma} \mapsto\left(e^{t \Lambda} x\right)^{\gamma}$ preserves the property (2.8), we see that $Q_{+} V$ is a finite sum of vector functions whose components satisfy (2.8).

Next we will show that $\left(L_{\Lambda_{S}}+c\right) M\left(Q_{+} V\right) \ll 0$. We note that every monomial $x^{\delta}$ which appears in $\left(e^{t \Lambda_{N}} x\right)^{\gamma}$ satisfies $\langle\lambda, \gamma\rangle=\langle\lambda, \delta\rangle$. Indeed, the map $x \mapsto e^{t \Lambda_{N}} x$ induces a linear upper (lower) triangular transformation among the components of $x$ corresponding to the same Jordan block. Because $\lambda_{i}$ 's coincide with each other for such components, we have the assertion. In view of the definition of $Q_{+}$, the condition $\langle\lambda, \gamma\rangle-\lambda_{j} \leq-c$ is preserved by the 
operator $Q_{+}$. Hence we have $Q_{+} V \in \mathcal{A}_{-}$. Therefore $Q_{+}: \mathcal{A}_{-} \mapsto \mathcal{A}_{-}$is well-defined if $\Lambda_{N}=0$. We remark that the above argument also shows that $Q_{+}: \mathcal{A}_{-}^{0} \mapsto \mathcal{A}_{-}^{0}$ is well-defined if $\Lambda_{N} \neq 0$.

Next we will show that $L_{\Lambda} Q_{+} V=V$ for $V \in \mathcal{A}_{-}$. For every $V \in \mathcal{A}_{-}$we will prove

$$
e^{-t \Lambda}\left\langle\Lambda x, \partial_{x}\right\rangle V\left(e^{t \Lambda} x\right)=e^{-t \Lambda} \frac{d}{d t} V\left(e^{t \Lambda} x\right), \quad t \geq 0,
$$

in some neighborhood of the origin $x=0$ independent of $t, 0 \leq t<\infty$. Indeed, by the relation $\partial_{x} V\left(e^{t \Lambda} x\right)=(\nabla V)\left(e^{t \Lambda} x\right) e^{t \Lambda}$ we have

$$
\begin{aligned}
\left\langle\Lambda x, \partial_{x}\right\rangle V\left(e^{t \Lambda} x\right) & =(\nabla V)\left(e^{t \Lambda} x\right) e^{t \Lambda} \Lambda x \\
& =(\nabla V)\left(e^{t \Lambda} x\right) \frac{d}{d t} e^{t \Lambda} x=\frac{d}{d t} V\left(e^{t \Lambda} x\right) .
\end{aligned}
$$

This proves (4.6) for each $t \geq 0$ and $x$ in some neighborhood of the origin. If $\Lambda_{N}=0$, then we have

$$
e^{-t \Lambda} \frac{d}{d t} V\left(e^{t \Lambda} x\right)=\sum_{\gamma}\langle\lambda, \gamma\rangle e^{t\langle\lambda, \gamma\rangle-\Lambda_{S} t} V_{\gamma} x^{\gamma}
$$

where $V(x)=\sum_{\gamma} V_{\gamma} x^{\gamma}$. Because $\left\|e^{t\langle\lambda, \gamma\rangle-\Lambda_{S} t}\right\| \leq 1$ for all $t \geq 0$, we see that the right-hand side is holomorphic in some neighborhood of $x=0$ independent of $t$. By an analytic continuation, (4.6) holds for all $x$ in some neighborhood of the origin independent of $t, t \geq 0$. This proves (4.6).

By (4.6) we have

$$
\begin{aligned}
L_{\Lambda} Q_{+} V & =\left(\left\langle\Lambda x, \partial_{x}\right\rangle-\Lambda\right) Q_{+} V \\
& =-\int_{0}^{\infty} e^{-t \Lambda}\left\langle\Lambda x, \partial_{x}\right\rangle V\left(e^{t \Lambda} x\right) d t+\Lambda \int_{0}^{\infty} e^{-t \Lambda} V\left(e^{t \Lambda} x\right) d t \\
& =-\int_{0}^{\infty} e^{-t \Lambda} \frac{d}{d t} V\left(e^{t \Lambda} x\right) d t+\Lambda \int_{0}^{\infty} e^{-t \Lambda} V\left(e^{t \Lambda} x\right) d t \\
& =-\int_{0}^{\infty} \frac{d}{d t}\left(e^{-t \Lambda} V\left(e^{t \Lambda} x\right)\right) d t=V(x) .
\end{aligned}
$$

Finally we shall prove the estimate. If $\Lambda$ is semi-simple, then we have

$$
Q_{+} V_{j}(x)=-\sum_{\gamma} x^{\gamma} \int_{0}^{\infty} \exp \left(t\langle\lambda, \gamma\rangle-t \lambda_{j}\right) d t V_{j, \gamma}=\sum_{\gamma} x^{\gamma}\left(\langle\lambda, \gamma\rangle-\lambda_{j}\right)^{-1} V_{j, \gamma} .
$$

Therefore, there exists $c_{1}>0$ independent of $V$ such that $\left\|Q_{+} V\right\|_{\rho} \leq c_{1}\|V\|_{\rho}$. This ends the proof.

For the later use, we give several lemmas. 
Lemma 4.2. If $f \in \mathbb{C}^{1}[x], g \in \mathbb{C}^{1}[x]$ and $c$ is a complex number, then $M(f g) \ll M(f) M(g), M(f+g) \ll M(f)+M(g)$ and $M(c f) \ll|c| M(f)$.

The proof is clear from the definition.

Lemma 4.3. Assume that $0 \ll f \ll g$ and $c>0$. If $\left(L_{\Lambda_{S}}+c\right) g \ll 0$, then $\left(L_{\Lambda_{S}}+c\right) f \ll 0$. Similarly, if $\left(L_{\Lambda_{S}}-c\right) g \gg 0$, then $\left(L_{\Lambda_{S}}-c\right) f \gg 0$.

Proof. Suppose that $\left(L_{\Lambda_{S}}+c\right) g \ll 0$. If $f=\sum f_{\gamma} x^{\gamma}$ and $g=\sum g_{\gamma} x^{\gamma}$, then $(\langle\lambda, \gamma\rangle+c) g_{\gamma} \leq 0$ and $0 \leq f_{\gamma} \leq g_{\gamma}$. If $g_{\gamma}=0$, then we have $f_{\gamma}=0$ and hence $(\langle\lambda, \gamma\rangle+c) f_{\gamma} \leq 0$. On the other hand, if $\langle\lambda, \gamma\rangle+c \leq 0$, then $(\langle\lambda, \gamma\rangle+c) f_{\gamma} \leq 0$. This proves that $\left(L_{\Lambda_{S}}+c\right) f \ll 0$. We can prove the latter half similarly.

Remark 4. The spaces $\mathcal{A}_{ \pm}$are linear spaces. Indeed, let $f, g \in \mathcal{A}_{-}$and $\alpha \in \mathbb{C}$. The condition (2.8) is easily verified for $f+g$ or $\alpha f$. We set $\mathcal{L}:=L_{\Lambda_{S}}+c$ $(c>0)$. Then $\mathcal{L} M(f) \ll 0$ and $\mathcal{L} M(g) \ll 0$ imply that $\mathcal{L}(M(f)+M(g)) \ll 0$. Because $M(f+g) \ll M(f)+M(g)$ by Lemma 4.2, it follows from Lemma 4.3 that $\mathcal{L} M(f+g) \ll 0$. This proves that $\mathcal{A}_{-}$is a linear space. The proof is the same for $\mathcal{A}_{+}$.

Lemma 4.4. Let $\rho>0$. For $u, v \in \mathbb{C}^{n}[x]$ such that $\|u\|_{\rho}<\infty$ and $\|v\|_{\rho}<\infty$, we have $\|u \cdot v\|_{\rho} \leq\|u\|_{\rho}\|v\|_{\rho}$.

This is clear from the definition.

\section{§5. Proof of Theorem 2.1}

Proof of Theorem 2.1. We will prove the theorem in the case $R \in \mathcal{A}_{-}$. The proof is the same in the case $R \in \mathcal{A}_{+}$. If there is no fear of confusion, we omit the suffices and we simply denote $\mathcal{A}$ and $Q$ instead of $\mathcal{A}_{\mp, \rho}$ and $Q_{ \pm, \rho}$, respectively. Similarly, we sometimes omit the suffix of $\|\cdot\|_{\rho}$ and write $\|\cdot\|$ instead of $\|\cdot\|_{\rho}$.

In order to solve (2.5) we set $v=Q V$. By Lemma 4.1, Eq. (2.5) can be written in the form

$$
V=R(x+Q V)
$$

if $\Lambda_{N}=0$. In view of this we will solve (5.1). We define the sequence $\left\{V^{j}\right\}_{j}$ by

$$
V^{0}=R(x), V^{1}=R\left(x+Q V^{0}\right)-R(x),
$$




$$
V^{j+1}=R\left(x+Q V^{0}+\cdots+Q V^{j}\right)-R\left(x+Q V^{0}+\cdots+Q V^{j-1}\right), j=1,2, \ldots
$$

In order to show that $V^{j}$ 's are well-defined we first consider the case $\Lambda_{N} \neq 0$. By Lemma 4.1 and the assumption, we see that $V^{0}$ and $Q V^{0}$ are polynomials. Hence, by (5.2) $V^{1}$ is a polynomial. Inductively, we see that $V^{j}$ 's are polynomials. We will show that $V^{j} \in \mathcal{A}^{0}$. For this purpose, we will prove that $R(x+Q V) \in \mathcal{A}^{0}$ if $V \in \mathcal{A}^{0}$. Indeed, if we can prove this, then we have $R\left(x+Q V^{0}\right) \in \mathcal{A}^{0}$. It follows that $V^{1}=R\left(x+Q V^{0}\right)-R(x) \in \mathcal{A}^{0}$. Next we have $V^{0}+V^{1} \in \mathcal{A}^{0}$, and thus $V^{2} \in \mathcal{A}^{0}$. By the induction we have $V^{j} \in \mathcal{A}^{0}$ $(j=0,1,2, \ldots)$.

In order to show (2.8) we write

$$
R_{j}(x+Q V)=\sum_{\gamma} R_{j, \gamma}(x+Q V)^{\gamma}=\sum_{\gamma} R_{j, \gamma} \prod_{i}\left(x_{i}+(Q V)_{i}\right)^{\gamma_{i}},
$$

where $(Q V)_{i}$ is the $i$-th component of $Q V$. Because $Q V \in \mathcal{A}^{0},(Q V)_{k}$ is the sum of the functions $h_{\mu}$ with $h_{\mu}$ being divisible by $x_{\mu}$ where $x_{k}$ and $x_{\mu}$ belong to the same Jordan block. If $R_{j}(x)$ is divisible by $x_{k}$ with $x_{k}$ and $x_{j}$ belonging to the same Jordan block, then it follows that $R_{j}(x+Q V)$ is the sum of functions divisible by some $x_{\nu}$ with $x_{\nu}$ and $x_{j}$ belonging to the same Jordan block. Hence (2.8) holds.

Next we will show that $\left(L_{\Lambda_{S}}+c\right) M(R(\cdot+Q V)) \ll 0$. Let $x^{\eta}$ be any monomial appearing in the right-hand side of (5.4). Because $\left(L_{\Lambda_{S}}+c\right) M(R) \ll$ 0 by the assumption, it follows that the $\gamma$ 's in (5.4) satisfy that $\langle\lambda, \gamma\rangle-\lambda_{j} \leq-c$. In view of the relation $Q V \in \mathcal{A}^{0},(Q V)_{i}$ can be expanded in the power series of $x^{\delta}$ such that $\langle\lambda, \delta\rangle-\lambda_{i} \leq-c$. If we expand $\left(x_{i}+(Q V)_{i}\right)^{\gamma_{i}}$ in the right-hand side of (5.4) into the power series of $x$, then we see that $x^{\eta}$ appears if some $x_{i}$ in $x^{\gamma}$ is replaced by $x^{\delta}$ appearing in $(Q V)_{i}$ a finite number of times. If $x^{\gamma}$ turns into $x^{\eta}$ by the one substitution, then we have

$$
\langle\lambda, \eta\rangle-\lambda_{j}=\langle\lambda, \gamma\rangle-\lambda_{j}-\lambda_{i}+\langle\lambda, \delta\rangle \leq-2 c<-c .
$$

By the similar argument, we have the estimate $\langle\lambda, \eta\rangle-\lambda_{j} \leq-c$ in the general case. Hence $R(x+Q V)$ satisfies $\left(L_{\Lambda_{S}}+c\right) M(R(\cdot+Q V)) \ll 0$. This proves that $R(x+Q V) \in \mathcal{A}^{0}$.

Next we consider the case $\Lambda_{N}=0$. Let $V \in \mathcal{A}$. Because $Q$ is continuous by Lemma 4.1, $R(x+Q V)$ is well-defined in some neighborhood of the origin if $\|V\|$ is sufficiently small. We will estimate $\|R(\cdot+Q V)\|_{\rho}$. By making the scale change of the variables, if necessary, one may assume $\|R\|_{2 \rho}<\varepsilon$. By Lemma 4.1, Lemma 4.4 and (4.1) we have

$$
\|R(\cdot+Q V)\|_{\rho} \leq \sum_{\gamma, j}\left|R_{j, \gamma}\right|\left(\rho+c_{1}\|V\|_{\rho}\right)^{|\gamma|} .
$$


If $\|V\|_{\rho}<\varepsilon$ for sufficiently small $\varepsilon$ such that $c_{1} \varepsilon<\rho$, then the right-hand side of (5.5) is bounded by $\varepsilon$ because $\|R\|_{2 \rho}<\varepsilon$. On the other hand, by the same argument as in the case $\Lambda_{N} \neq 0$, we can prove that $R(x+Q V) \in \mathcal{A}$. Therefore, one can define $V^{j} \in \mathcal{A}(j=0,1, \ldots)$ by (5.2) and (5.3) inductively.

Next we will prove the convergence of $\left\{V^{j}\right\}$ in $\mathcal{A}$. For this purpose we will show that there exist constants $c_{0} \geq 0$ and $K_{0} \geq 0$ independent of $j$ such that, for $j=0,1,2, \ldots$,

$$
\begin{gathered}
\left\|V^{j}\right\| \leq c_{0}^{j} \varepsilon^{j+1}, \\
\left\|Q V^{j}\right\| \leq K_{0}\left\|V^{j}\right\| .
\end{gathered}
$$

Clearly we have $\left\|V^{0}\right\|=\|R\|<\varepsilon$ by the definition. Next we will show (5.7) for $j=0$. If $\Lambda_{N}=0$, then the estimate follows from Lemma 4.1. Hence we may assume $\Lambda_{N} \neq 0$. Let $d_{0}$ be the degree of $R$. By Lemma 4.1 we have $Q V^{0} \in \mathcal{A}^{0}$. By the definition we have

$$
Q V^{0}=-\int_{0}^{\infty} e^{-t \Lambda} V^{0}\left(e^{t \Lambda} x\right) d t=-\int_{0}^{\infty} e^{-t \Lambda_{N}} e^{-t \Lambda_{S}} V^{0}\left(e^{t \Lambda} x\right) d t
$$

We set

$$
W(t, x) \equiv\left(W_{1}(t, x), \ldots, W_{n}(t, x)\right):=e^{-t \Lambda_{S}} V^{0}\left(e^{t \Lambda} x\right)
$$

Then the components of the first Jordan block of $e^{-t \Lambda_{N}} W$ are given by

$$
\begin{array}{r}
W_{1}-t W_{2}+\frac{t^{2}}{2} W_{3}-\frac{t^{3}}{3 !} W_{4}+\cdots \\
W_{2}-t W_{3}+\frac{t^{2}}{2 !} W_{4}-\cdots
\end{array}
$$

There are finite number of similar terms corresponding to every Jordan block of $e^{-t \Lambda_{N}} W$. Hence we can easily see that $\left\|Q V^{0}\right\|_{\rho}$ is bounded by the following 
quantity

$$
\begin{aligned}
& \sum_{k=0}^{n-1}\left\|\int_{0}^{\infty} \frac{(-t)^{k}}{k !} W_{k+1}(t, x) d t\right\|+\sum_{k=0}^{n-2}\left\|\int_{0}^{\infty} \frac{(-t)^{k}}{k !} W_{k+2}(t, x) d t\right\|+\cdots \\
\leq & \sum_{k=0}^{n-1} \int_{0}^{\infty} \frac{t^{k}}{k !}\left\|W_{k+1}(t, \cdot)\right\| d t+\sum_{k=0}^{n-2} \int_{0}^{\infty} \frac{t^{k}}{k !}\left\|W_{k+2}(t, \cdot)\right\| d t+\cdots \\
= & \int_{0}^{\infty}\left\|W_{1}(t, \cdot)\right\| d t+\int_{0}^{\infty}\left\|W_{2}(t, \cdot)\right\|(1+t) d t \\
+ & \int_{0}^{\infty}\left\|W_{3}(t, \cdot)\right\|\left(1+t+\frac{t^{2}}{2}\right) d t+\cdots \\
\leq & \sum_{j=1}^{n} \int_{0}^{\infty} B_{0}(t)\left\|W_{j}(t, \cdot)\right\| d t
\end{aligned}
$$

where $B_{0}(t)=\sum_{\nu=0}^{n-1} t^{\nu} / \nu$ !. We write $V^{0}=\left(V_{1}^{0}, V_{2}^{0}, \ldots, V_{n}^{0}\right)$, and expand $V_{j}^{0}$ $(1 \leq j \leq n)$ into the Taylor series $V_{j}^{0}=\sum_{\gamma} V_{j, \gamma}^{0} x^{\gamma}$. Then, by (5.9) we have

$$
\begin{aligned}
W_{j}(t, x) & =e^{-t \lambda_{j}} V_{j}^{0}\left(e^{t \Lambda} x\right)=e^{-t \lambda_{j}} \sum_{\gamma} V_{j, \gamma}^{0}\left(e^{t \Lambda} x\right)^{\gamma} \\
& =\sum_{\gamma} e^{t\langle\lambda, \gamma\rangle-t \lambda_{j}} V_{j, \gamma}^{0}\left(e^{t \Lambda_{N}} x\right)^{\gamma} .
\end{aligned}
$$

Because the sum with respect to $\gamma$ is finite, we have

$$
\sum_{j=1}^{n} \int_{0}^{\infty} B_{0}(t)\left\|W_{j}(t, \cdot)\right\| d t \leq \sum_{j, \gamma}\left|V_{j, \gamma}^{0}\right| \int B_{0}(t) e^{t\langle\lambda, \gamma\rangle-t \lambda_{j}}\left\|\left(e^{t \Lambda_{N}} x\right)^{\gamma}\right\|_{\rho} d t .
$$

On the other hand, we have

$$
\left\|\left(e^{t \Lambda_{N}} x\right)^{\gamma}\right\|_{\rho} \leq \rho^{|\gamma|}\left(e^{t \Lambda_{N}} \vec{e}\right)^{\gamma} \leq \rho^{|\gamma|} \sum_{|\gamma| \leq d_{0}}\left(e^{t \Lambda_{N}} \vec{e}\right)^{\gamma},
$$

where $\vec{e}=(1,1, \ldots, 1)$. In terms of the estimate $\langle\lambda, \gamma\rangle-\lambda_{j} \leq-c(j=$ $1,2, \ldots, n)$ and $(5.14)$, the right-hand side of $(5.13)$ can be estimated in the following way

$$
\begin{aligned}
& \leq \sum_{j, \gamma} \rho^{|\gamma|}\left|V_{j, \gamma}^{0}\right| \int B_{0}(t) e^{-c t} \sum_{|\delta| \leq d_{0}}\left(e^{t \Lambda_{N}} \vec{e}\right)^{\delta} d t \\
& \leq K\left(d_{0}\right) \sum_{j, \gamma} \rho^{|\gamma|}\left|V_{j, \gamma}^{0}\right|=K\left(d_{0}\right)\left\|V^{0}\right\|_{\rho}
\end{aligned}
$$


where $K\left(d_{0}\right)=\int B_{0}(t) e^{-c t} \sum_{|\delta| \leq d_{0}}\left(e^{t \Lambda_{N}} \vec{e}\right)^{\delta} d t$. Therefore we have $\left\|Q V^{0}\right\|_{\rho} \leq$ $K\left(d_{0}\right)\left\|V^{0}\right\|_{\rho}$. If we set $K_{0}=\max \left\{K\left(d_{0}\right), c_{1}\right\}$ with $c_{1}$ given by Lemma 4.1 , then we have (5.7) for $j=0$.

Next we will prove (5.6) for $j=1$. It follows from (5.2) that

$$
\left\|V^{1}\right\| \leq\left\|Q V^{0}\right\| \int_{0}^{1}\left\|\nabla R\left(\cdot+\tau Q V^{0}\right)\right\| d \tau .
$$

In order to estimate $\left\|\nabla R\left(\cdot+\tau Q V^{0}\right)\right\|$ we make the same argument as in (5.5). Indeed, if we have the estimate

$$
\sum_{\gamma}\left|R_{j, \gamma}\right||\gamma|(2 \rho)^{|\gamma|}<c_{2} \varepsilon, \quad j=1,2, \ldots, n,
$$

for some constant $c_{2}>0$ independent of $\varepsilon$, then we obtain

$$
\left\|\nabla R\left(\cdot+\tau Q V^{0}\right)\right\|<c_{2} \varepsilon, \forall \tau, 0 \leq \tau \leq 1,
$$

if $K\left(d_{0}\right) \varepsilon<\rho$. The estimate (5.17) follows from the assumption $\|R\|_{2 \rho}<\varepsilon$ if we replace $\rho>1$ with $1<\rho^{\prime}<\rho$. For the sake of simplicity we assume that (5.17) holds in the following.

Therefore we get, from (5.16) that

$$
\left\|V^{1}\right\| \leq K\left(d_{0}\right) c_{2} \varepsilon^{2} \int_{0}^{1} d \tau=c_{0} \varepsilon^{2},
$$

where $c_{0}=K\left(d_{0}\right) c_{2}$.

Next we will estimate $\left\|Q V^{1}\right\|$. In view of Lemma 4.1 we may assume that $\Lambda_{N} \neq 0$. We write $V^{1}=\left(V_{1}^{1}, V_{2}^{1}, \ldots, V_{n}^{1}\right)$ and consider the Taylor expansion $V_{j}^{1}(x)=\sum_{\gamma} V_{j, \gamma}^{1} x^{\gamma}$. Here the sum is a finite one. We will show that for every $\gamma$ such that $V_{j, \gamma}^{1} \neq 0$ we have

$$
\langle\lambda, \gamma\rangle-\lambda_{j} \leq-\frac{c}{d_{0}-1}(|\gamma|-1)
$$

Noting that $V_{j}^{1}=R_{j}(x+Q R)-R_{j}(x)$, we first consider $R_{j}(x)$. Because $R \in \mathcal{A}^{0}$, we have $\langle\lambda, \gamma\rangle-\lambda_{j} \leq-c(j=1,2, \ldots, n)$ for every $x^{\gamma}$ in the expansion of $R_{j}(x)$. Because $|\gamma| \leq d_{0}$, we have

$$
\langle\lambda, \gamma\rangle-\lambda_{j} \leq-c=-c \frac{d_{0}-1}{d_{0}-1} \leq-\frac{c}{d_{0}-1}(|\gamma|-1), \quad j=1,2, \ldots, n .
$$

Next we will prove (5.20) for $R_{j}(x+Q R)$. We note that $Q R$ satisfies (5.20) because $Q R \in \mathcal{A}^{0}$ and $Q R$ is the polynomial of degree $d_{0}$. We set 
$z=x+Q R$ and we consider $R_{j}(z)=\sum_{\delta} R_{j, \delta} z^{\delta}=\sum_{\gamma} \tilde{R}_{j, \gamma} x^{\gamma}$, where we expand $z^{\delta}=(x+Q R)^{\delta}$ into the polynomial of $x$ in the right-hand side. Then $x^{\gamma}$ appears in the expansion of $R_{j}(x+Q R)$ in the following way. In the term $x^{\delta}$, some $x_{j(1)}(1 \leq j(1) \leq n)$ is replaced by $x^{\delta^{(1)}}$ which appears in the expansion of $(Q R)_{j(1)}$, where $(Q R)_{j(1)}$ is the $j(1)$-th component of $Q R$. Next some $x_{j(2)}$ $(1 \leq j(2) \leq n)$ is replaced by $x^{\delta^{(2)}}$ which appears in the expansion of $(Q R)_{j(2)}$. In the same way, some $x_{j(k)}(1 \leq j(k) \leq n)$ is replaced by $x^{\delta^{(k)}}$ which appears in the expansion of $(Q R)_{j(k)},(k=1,2, \ldots, \nu)$. It follows that $\gamma$ has the expression

$$
\gamma=\delta+\delta^{(1)}-e_{j(1)}+\delta^{(2)}-e_{j(2)}+\cdots+\delta^{(\nu)}-e_{j(\nu)} .
$$

By noting that $Q R \in \mathcal{A}^{0}$ and by the same calculations as in (5.21) we have

$$
\begin{gathered}
\langle\lambda, \delta\rangle-\lambda_{j} \leq-c \leq-\frac{c}{d_{0}-1}(|\delta|-1), \\
\left\langle\lambda, \delta^{(k)}\right\rangle-\lambda_{j(k)} \leq-c \leq-\frac{c}{d_{0}-1}\left(\left|\delta^{(k)}\right|-1\right), \quad 1 \leq k \leq \nu .
\end{gathered}
$$

Hence, by (5.22) we have

$$
\begin{aligned}
& \langle\lambda, \gamma\rangle-\lambda_{j}=\langle\lambda, \delta\rangle-\lambda_{j}+\sum_{k=1}^{\nu}\left(\left\langle\lambda, \delta^{(k)}\right\rangle-\lambda_{j(k)}\right) \\
& \leq-\frac{c}{d_{0}-1}\left(|\delta|-1+\sum_{k=1}^{\nu}\left(\left|\delta^{(k)}\right|-1\right)\right)=-\frac{c}{d_{0}-1}(|\gamma|-1), \quad j=1,2, \ldots, n .
\end{aligned}
$$

This proves (5.20).

We will estimate $\left\|Q V^{1}\right\|$. By the same argument as for $\left\|Q V^{0}\right\|$ we have

$$
\begin{aligned}
\left\|Q V^{1}\right\|_{\rho} & \leq \sum_{j, \gamma}\left|V_{j, \gamma}^{1}\right| \int_{0}^{\infty} B_{0}(t) e^{t\langle\lambda, \gamma\rangle-t \lambda_{j}}\left\|\left(e^{t \Lambda_{N}} x\right)^{\gamma}\right\|_{\rho} d t \\
& =\sum_{j,|\gamma| \geq \nu_{0}}+\sum_{j,|\gamma|<\nu_{0}} \equiv I_{2}+I_{1},
\end{aligned}
$$

where $V^{1}=\sum_{\gamma} V_{j, \gamma}^{1} x^{\gamma}$ and $\nu_{0}$ is a sufficiently large integer chosen later. We consider $I_{1}$. We have

$$
\left\|\left(e^{t \Lambda_{N}} x\right)^{\gamma}\right\|_{\rho} \leq \rho^{|\gamma|} \times\left(\text { a polynomial of } t \text { with degree determined by } \nu_{0}\right) .
$$

On the other hand, we have $\langle\lambda, \gamma\rangle-\lambda_{j} \leq-c$ and $c>d_{0}-1 \geq 1$ by the assumption. It follows that $I_{1}$ can be bounded by

$$
I_{1} \leq \sum_{j,|\gamma|<\nu_{0}}\left|V_{j, \gamma}^{1}\right| \rho^{|\gamma|} K_{1},
$$


where $K_{1}$ depends on $\nu_{0}$.

Next we consider $I_{2}$. By the definitions of the norm and $e^{t \Lambda_{N}}$ we have

$$
\left\|\left(e^{t \Lambda_{N}} x\right)^{\gamma}\right\|_{\rho} \leq \rho^{|\gamma|}\left(e^{t \Lambda_{N}} \vec{e}\right)^{\gamma} \leq \rho^{|\gamma|} e^{t|\gamma|}
$$

where $\vec{e}={ }^{t}(1,1, \ldots, 1)$. Hence, by (5.20) we have

$$
I_{2} \leq \sum_{j,|\gamma| \geq \nu_{0}}\left|V_{j, \gamma}^{1}\right| \rho^{|\gamma|} \int_{0}^{\infty} B_{0}(t) \exp \left(\frac{c t}{d_{0}-1}-\frac{c t|\gamma|}{d_{0}-1}+t|\gamma|\right) d t .
$$

By the assumption $c>d_{0}-1$ there exists $\kappa>0$ such that $\left(c /\left(d_{0}-1\right)-1\right)>\kappa$. We take $\nu_{0}$ so that $c /\left(d_{0}-1\right)<\kappa \nu_{0}$. Then we have

$$
\frac{c t}{d_{0}-1}-\frac{c t|\gamma|}{d_{0}-1}+t|\gamma| \leq \frac{c t}{d_{0}-1}-\kappa t|\gamma| \leq t\left(\frac{c}{d_{0}-1}-\kappa \nu_{0}\right) .
$$

Hence the integral in the right-hand side of (5.29) converges and it is bounded by some constant $K_{2}$ independent of $\gamma$. Therefore we have

$$
I_{2} \leq K_{2} \sum_{j, \gamma}\left|V_{j, \gamma}^{1}\right| \rho^{|\gamma|} .
$$

We note that $K_{1}$ and $K_{2}$ depend on $c, d_{0}$ and the dimension $n$, and are independent of $\gamma$. By (5.26), (5.27) and (5.30) we have

$$
\left\|Q V^{1}\right\|_{\rho} \leq I_{2}+I_{1} \leq K_{0}\left\|V^{1}\right\|_{\rho}
$$

where $K_{0}=\max \left\{K_{1}+K_{2}, K\left(d_{0}\right), c_{1}\right\}$ with $c_{1}$ given by Lemma 4.1. This proves (5.7) for $j=1$.

We will prove (5.6) and (5.7) by induction. Suppose that we have proven (5.6) and (5.7) for $j=0,1,2, \ldots, k$. Then, by (5.6) and (5.7) we have

$$
\left\|Q\left(V^{0}+\cdots+V^{k}\right)\right\| \leq K_{0} \sum_{\nu=0}^{k}\left\|V^{\nu}\right\| \leq K_{0} \varepsilon \sum_{\nu=0}^{k}\left(\varepsilon c_{0}\right)^{\nu}=2 K_{0} \varepsilon,
$$

if $\varepsilon c_{0}<1 / 2$. Hence, if $\rho+2 K_{0} \varepsilon<2 \rho$, then $V^{k+1}$ is well-defined. Moreover, by the definition we have

$$
\begin{aligned}
\left\|V^{k+1}\right\| & \leq\left\|Q V^{k}\right\| \int_{0}^{1}\left\|\nabla R\left(\cdot+Q V^{0}+\cdots+Q V^{k-1}+\tau Q V^{k}\right)\right\| d \tau \\
& \leq K_{0} c_{0}^{k} \varepsilon^{k+1} c_{2} \varepsilon=c_{0}^{k+1} \varepsilon^{k+2}
\end{aligned}
$$

where $c_{0}=K_{0} c_{2}$. Here we used the estimate

$$
\left\|\nabla R\left(\cdot+Q V^{0}+\cdots+Q V^{k-1}+\tau Q V^{k}\right)\right\|<c_{2} \varepsilon
$$


which follows by the similar argument like (5.18).

Next we will prove (5.7) for $j=k+1$. If $\Lambda_{N}=0$, then the assertion follows from Lemma 4.1. Hence we assume $\Lambda_{N} \neq 0$. Let $V^{k+1}={ }^{t}\left(V_{1}^{k+1}, \ldots, V_{n}^{k+1}\right)$ and let $V_{j}^{k+1}(x)=\sum_{\gamma} V_{j, \gamma}^{k+1} x^{\gamma},(j=1,2, \ldots, n)$ be the Taylor expansion. First we will prove $(5.20)$ for $\gamma$ such that $V_{j, \gamma}^{k+1} \neq 0$. We will prove this by the induction on $\nu$ of $V_{j}^{\nu}$. Suppose that $(5.20)$ holds for $V_{j}^{\nu},(\nu=0,1, \ldots, m)$ $(m \leq k)$ in such a way that $R_{j}\left(x+Q V^{0}+\cdots+Q V^{\nu}\right)(\nu=0,1, \ldots, m-1)$ also satisfy (5.20). We have already proved the assertion for $m=0,1$. We want to show that $R_{j}\left(x+Q V^{0}+\cdots+Q V^{m}\right)(1 \leq j \leq m)$ satisfies (5.20). If we can prove this, then $V_{j}^{m+1}$ satisfies (5.20) by the inductive assumption and the definition of $V_{j}^{m+1}$.

We note that every $j$-th component of $Q V^{\nu}(\nu=0,1, \ldots, m)$ also satisfies (5.20). Indeed, $Q$ maps every monomial vector $x^{\gamma} W_{\gamma}\left(W_{\gamma}\right.$, a constant vector) to the vector whose components are the polynomials of the form $\sum_{\langle\lambda, \gamma\rangle=\langle\lambda, \delta\rangle} c_{\delta} x^{\delta}$ for some constants $c_{\delta}$. Hence, in view of the definition of $Q$, we have the assertion. Let

$$
R_{j}(z)=\sum_{\delta} R_{j, \delta} z^{\delta}=\sum_{\gamma} \tilde{R}_{j, \gamma} x^{\gamma}
$$

be the Taylor expansion at the origin, where $z=x+Q V^{0}+\cdots+Q V^{m}$. For every $\nu$ and $j,(0 \leq \nu \leq m, j=1,2, \ldots, n)$, we denote by $\left(Q V^{\nu}\right)_{j}$ the $j$-th component of $Q V^{\nu}$. If $x^{\gamma}$ appears in the expansion of $z^{\delta}=\left(x+Q V^{0}+\cdots+Q V^{m}\right)^{\delta}$, then we see that some $x_{j(1)}(1 \leq j(1) \leq n)$ in the monomial $x^{\delta}$ is replaced by $x^{\delta^{(1)}}$ which appears in the expansion of some $\left(Q V^{\nu}\right)_{j(1)},(0 \leq \nu \leq m)$. Similarly, $x_{j(2)}(1 \leq j(2) \leq n)$ in the monomial $x^{\delta}$ is replaced by some $\left(Q V^{\nu}\right)_{j(2)}$ and so on. Hence we see that (5.22) holds.

Because $\delta^{(k)}$ satisfies (5.20) by the inductive assumption we have

$$
\left\langle\lambda, \delta^{(k)}\right\rangle-\lambda_{j(k)}=\left\langle\lambda, \delta^{(k)}-e_{j(k)}\right\rangle \leq-\frac{c}{d_{0}-1}\left(\left|\delta^{(k)}\right|-1\right) .
$$

Summing up with respect to $k$ and by using $|\gamma|=|\delta|+\sum_{k}\left(\left|\delta^{(k)}\right|-1\right)$ which follows from (5.22) we have

$$
\begin{aligned}
\langle\lambda, \gamma\rangle-\lambda_{j} & =\langle\lambda, \delta\rangle-\lambda_{j}+\sum_{k=0}^{\nu}\left\langle\lambda, \delta^{(k)}-e_{j(k)}\right\rangle \\
& \leq-\frac{c}{d_{0}-1}\left(|\delta|-1+\sum_{k}\left(\left|\delta^{(k)}\right|-1\right)\right)=-\frac{c}{d_{0}-1}(|\gamma|-1)
\end{aligned}
$$

Hence we have proved that $R_{j}\left(x+Q V^{0}+\cdots+Q V^{m}\right)(1 \leq j \leq m)$ satisfies (5.20). By induction (5.20) holds for $\gamma$ such that $V_{j, \gamma}^{k+1} \neq 0$. If we can prove 
(5.20), then the remaining argument of the proof of (5.7) for $j=k+1$ is identical with the one for $\left\|Q V^{1}\right\|$.

By (5.6) we see that the limit $V:=\sum_{j=0}^{\infty} V^{j}$ exists in $\mathcal{A}$. If $\Lambda_{N}=0$, then the domain of $Q$ contains $\mathcal{A}$ and satisfies $L_{\Lambda} Q=I d$ on $\mathcal{A}$ by Lemma 4.1. It follows that $v:=Q V$ is a solution of (5.1).

Next we consider the case $\Lambda_{N} \neq 0$. Because $V^{0}=O\left(|x|^{2}\right)$ by the assumption, we see from (5.2) that $V^{1}=O\left(|x|^{3}\right)$. Inductively, we can see that $V^{j}=O\left(|x|^{j+2}\right)$ for $j=0,1,2, \ldots$. It follows that for every $x^{\gamma}$, the coefficient of $x^{\gamma}$ in $V=\sum_{0}^{\infty} V^{j}$ is a finite sum. Because every $V^{j}$ satisfies (5.20), it follows that every component of $V$ satisfies (5.20).

We will show that $Q V$ is well-defined. We set $V=\left(V_{1}, V_{2}, \ldots, V_{n}\right)$. Let $V_{j}=\sum_{\gamma} V_{j, \gamma} x^{\gamma}$ be the Taylor expansion at the origin and consider

$$
\begin{aligned}
e^{-t \Lambda} V\left(e^{t \Lambda} x\right) & =e^{-t \Lambda}\left(V_{j}\left(e^{t \Lambda} x\right)\right)_{j} \\
& =e^{-t \Lambda_{N}}\left(\sum_{\gamma} e^{t\langle\lambda, \gamma\rangle-t \lambda_{j}} V_{j, \gamma}\left(e^{t \Lambda_{N}} x\right)^{\gamma}\right)_{j}
\end{aligned}
$$

By integrating both sides of (5.36) we make the same argument as in the estimate of $\left\|Q V^{1}\right\|$ in (5.26), (5.27), (5.28) and (5.29). Indeed, we have (5.26) with $V_{j, \gamma}^{1}$ replaced by $V_{j, \gamma}$. Then we can show the estimates (5.27) and (5.30). Hence $Q V$ is well-defined. Moreover, the argument shows that the sum in the right-hand side of (5.36) converges uniformly in $t, 0 \leq t<\infty$ and in $x$ when $x$ is in some neighborhood of the origin.

Next we will prove $L_{\Lambda} Q V=V$. We make the same argument as in (4.8). Indeed, it is sufficient to show that (4.6) holds for all $x$ in some neighborhood of the origin independent of $t$. First, by (4.7) we have that, for each $t,\left\langle\Lambda x, \partial_{x}\right\rangle V\left(e^{t \Lambda} x\right)=\frac{d}{d t} V\left(e^{t \Lambda} x\right)$ in some neighborhood of the origin $x=0$ possibly depending on $t$. On the other hand, we have

$$
\begin{aligned}
& e^{-t \Lambda} \frac{d}{d t} V\left(e^{t \Lambda}\right)=e^{-t \Lambda} \frac{d}{d t} \sum_{\gamma} e^{t\langle\lambda, \gamma\rangle} V_{\gamma}\left(e^{t \Lambda_{N}} x\right)^{\gamma} \\
= & e^{-t \Lambda_{N}} \sum_{\gamma} e^{t\langle\lambda, \gamma\rangle-t \Lambda_{S}} V_{\gamma}\left(\langle\lambda, \gamma\rangle\left(e^{t \Lambda_{N}} x\right)^{\gamma}+\frac{d}{d t}\left(e^{t \Lambda_{N}} x\right)^{\gamma}\right) .
\end{aligned}
$$

By applying the same argument as the one in showing the uniform convergence of (5.36) we can show that the right-hand side of (5.37) is an analytic function of $x$ in some neighborhood of the origin independent of $t, 0 \leq t<\infty$. Therefore, by the analytic continuation (4.6) holds in some neighborhood of the origin $x=0$ independent of $t$. Hence we have $L_{\Lambda} Q V=V$. This proves that $v:=Q V$ is a solution of (2.2), which ends the proof of Theorem 2.1. 


\section{Acknowledgement}

The author would like to express his gratitude to the anonymous referee for pointing out critical remarks.

\section{References}

[1] H. Ito, Integrability of Hamiltonian systems and Birkhoff normal forms in the simple resonance case, Math. Ann. 292 (1992), no. 3, 411-444.

[2] R. Pérez-Marco, Total convergence or general divergence in small divisors, Comm. Math. Phys. 223 (2001), no. 3, 451-464.

[3] W. M. Schmidt, Diophantine approximation, Lecture Notes in Math., 785, Springer, Berlin, 1980.

[4] L. Stolovitch, Singular complete integrability, Inst. Hautes Études Sci. Publ. Math. No. 91 (2000), 133-210.

[5] N. T. Zung, Convergence versus integrability in Poincaré-Dulac normal form, Math. Res. Lett. 9 (2002), no. 2-3, 217-228. 